Cinémas

Revue d'études cinématographiques

Journal of Film Studies

\title{
Le Droit du plus fort
}

\section{Alain-N. Moffat}

Volume 1, numéro 1-2, automne 1990

Américanité et cinéma

URI : https://id.erudit.org/iderudit/1000995ar

DOI : https://doi.org/10.7202/1000995ar

Aller au sommaire du numéro

Éditeur(s)

Cinémas

ISSN

1181-6945 (imprimé)

1705-6500 (numérique)

Découvrir la revue

Citer cet article

Moffat, A.-N. (1990). Le Droit du plus fort. Cinémas, 1(1-2), 104-111.

https://doi.org/10.7202/1000995ar

\section{Résumé de l'article}

Analysant cinq films de l'ONF qui ont précisément pour thème "L'Américanité », l'auteur dénote ici deux visions concurrentes de la société québécoise et des rapports qu'elle entretient avec le temps. D’une part une vision pessimiste de l'Histoire comme chronologie fatale entre le passé et l'avenir, d'autre part un travail de la mémoire tout entier voué au présent et à la dissolution du chronologique. 


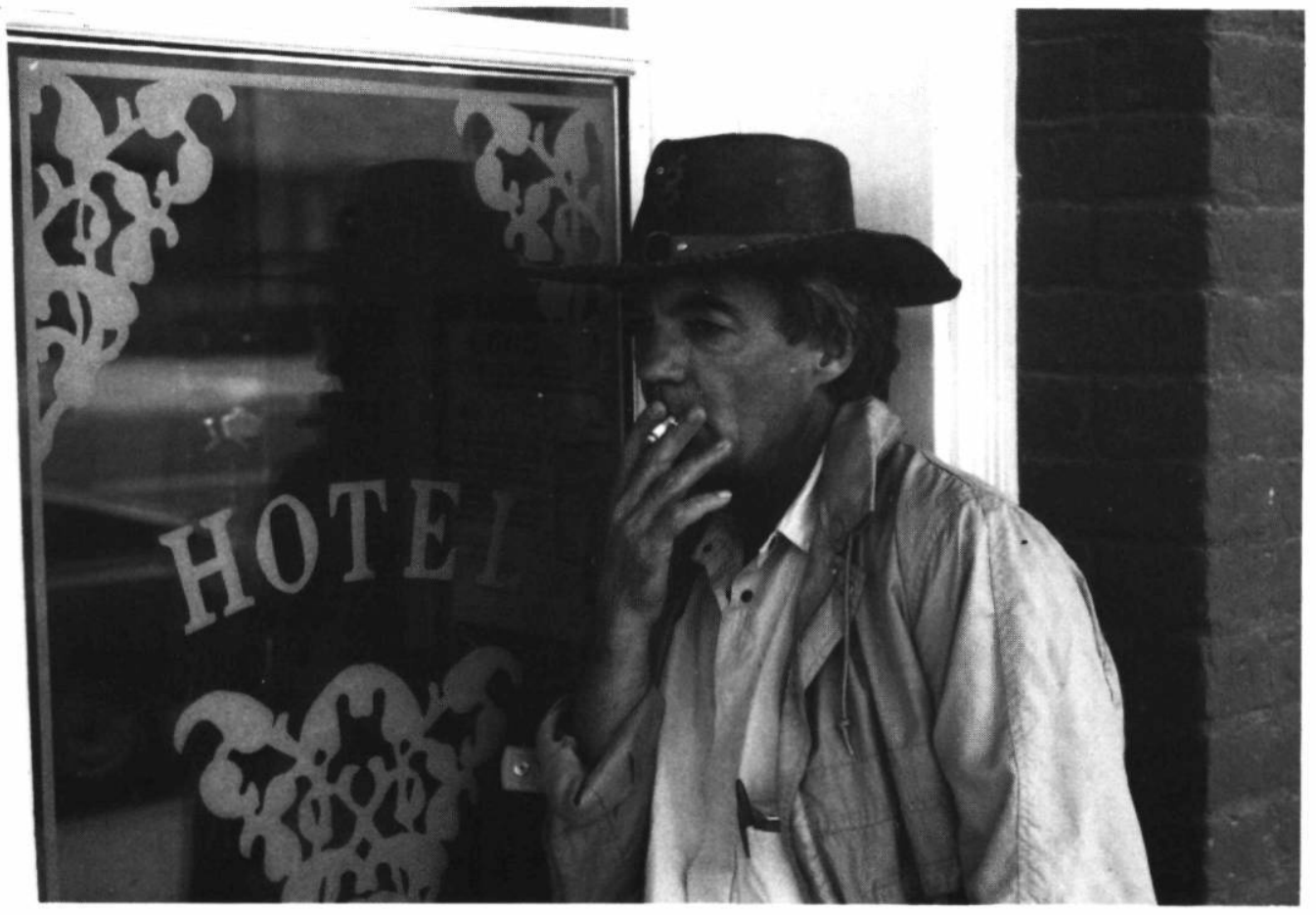

Alias Will James de Jacques Godbout (1988) Coll. ONF 


\title{
Le Droit du plus fort
}

\section{Alain-N. Moffat}

\section{RÉSUMÉ}

Analysant cing films de l'ONF qui ont précisément pour thème "L'Américanité», l'auteur dénote ici deux visions concurrentes de la société québécoise et des rapports qu'elle entretient avec le temps. D'une part une vision pessimiste de l'Histoire comme chronologie fatale entre le passé et l'avenir, d'autre part un travail de la mémoire tout entier voué au présent et à la dissolution du chronologique.

\begin{abstract}
Through an analysis of the films in the "L'Américanité» series from National Film Board, the author presents two conflicting views of Quebec society and its relationship with Time. On one hand, the pessimistic view regards History as an foreordained chronology linking the past to the future. On the other, History is seen as the working of memory as a whole, dedicated to the present and to the dissolution of chronology.
\end{abstract}

"L'Amérique n'a pas compris le montage, l'Amérique reste narrative.» Sergeï M. Eisenstein Mémoires

«(...) je suppose que dans toute société la production du discours est à la fois contrôlée, sélectionnée, organisée et redistribuée par un certain nombre de procédures qui ont pour rôle d'en conjurer les pouvoirs et les dangers, d'en maîtriser l'événement aléatoire, d'en esquiver la lourde, la redoutable matérialité.» Michel Foucault L'Ordre du discours

À l'heure du libre-échange, de la culture d'entreprise et de la mondialisation des marchés, l'Office national du film produit une 
série de films qui a pour thème l'impact de l'Amérique sur l'identité nationale. On décide de lui donner le titre «L'Américanité». Le mot est intéressant. Le mot est significatif. D'une part, en choisissant de parler d'«américanité» plutôt que d'idéologie américaine, on cherche à assigner à l'Amérique tous les traits et toutes les valeurs d'une culture; d'autre part, c'est précisément en limitant cette série à la seule «société distincte» et à ses représentants au sein de l'Office que l'on peut donner à l'Amérique toute sa dimension culturelle. Il faut donc reconnaître que cette entreprise nous renseigne beaucoup plus sur ce que nous sommes, sur notre «québécitude», que sur l'Amérique elle-même.

Nous savons pourtant que la lutte contre le libre-échange, qui est une lutte fondamentale contre la réification des hommes et des femmes et pour la survie des différences, pour la culture donc, n'est pas venue du Québec et qu'elle ne viendra pas du Québec. À défaut de s'identifier à la fédération canadienne, notre empathie américaine pourrait bien dépasser notre volonté d'indépendance et le pays réel que nous avions souhaité pourrait bien être remplacé par celui, nihiliste et fatal, du grand vide américain.

Octavio Paz définit bien l'Amérique par cette formule: «L'utopie américaine est un mélange de trois rêves: celui de l'ascète, celui du marchand et celui de l'explorateur. Trois individualistes» (p. 49-50). Nous avons déjà connu l'explorateur: au $\mathrm{XIX}^{\mathrm{e}}$ siècle, il a échoué lamentablement sur les rives de la fédération canadienne. Il nous restait à connaître le repli sur soi de l'ascète et le triomphe des intérêts particuliers du marchand. Maintenant que le discours économique emprunte les apparences de la démocratie, qu'il fait tomber les murs et entraîne tout le monde dans les centres d'achat, que la culture marchande engouffre toutes les aspirations populaires, que les peuples et les individus compétitionnent avec les marchandises, à quoi pourrait bien ressembler la culture d'une société dite distincte?

Et si dans ce monde une culture uniforme et sans histoire, une culture du même et de la série prenait le pas sur l'affirmation des différences, que resterait-il du désir ? Viviane Forrester n'écritelle pas déjà:

(...)l'horreur économique évoquée par Rimbaud sous-tend chaque parcelle du temps, de l'espace, inaugure les systèmes, accapare la langue, camoufle les langages, se donne pour phénomène naturel antérieur à tous les temps. Se fond avec la religion, s'infiltre dans l'environnement, codifie la morale. Crée des catégories (p. 15).

Si la culture est inséparable de l'histoire du présent, de l'histoire qui se fait, en abordant le thème de l'«américanité» et la série de l'ONF, il nous faut reconnaître - comme le fait si justement 
Octavio Paz - que les Etats-Unis d'Amérique sont un pays fondé avant tout contre l'Europe, contre la culture et contre l'histoire. Sans cesse enivrée par la tyrannie du nouveau, de l'excellence et du futur, l'Amérique trouve son point d'appui dans l'idée d'un progrès démesuré où «le bien commun ne réside pas dans une finalité collective ou métahistorique, mais dans la coexistence harmonieuse des fins individuelles» (Paz, p. 42). D'où cette idée de recherche du bonheur qui est inscrite dans la Déclaration d'indépendance américaine et qui constitue le thème à partir duquel se construit le film de Micheline Lanctôt, La Poursuite du bonheur (1987). Il n'est pas sans intérêt par ailleurs de noter que cette idée plutôt abstraite, parce que détachée du présent, fut inscrite à la toute dernière minute dans la Déclaration d'indépendance, en remplacement de celle, beaucoup plus révélatrice, du droit à la propriété privée.

Le portrait de la société de consommation tracé par Micheline Lanctôt est sans pitié. La quête du bonheur se transforme en quête des signes de l'aliénation des masses à la société de consommation. Mais la forme du film est désordonnée: les exemples de l'aliénation se multiplient sans subtilités et sans jamais en souligner la cause. Des stratégies de mise en marché au mode de production et de consommation du poulet, en passant par l'«insipide» André Moreau, la ville de Laval et son idéologie familiale, tout est tellement gros qu'à la fin, lorsque la réalisatrice désigne le «système» comme grand responsable, celui-ci demeure toujours aussi abstrait, comme une espèce de fatalité flottant au-dessus de nos têtes. C'est qu'ici, semble-t-il, on hésite à dire que ce système est capitaliste, qu'il est basé sur l'exploitation des ressources humaines et naturelles, sur l'appropriation par une minorité de la majorité des richesses et que ce processus en s'accélérant aliène de plus en plus les individus et polarise de plus en plus les classes sociales. Il n'est pas sans intérêt d'ailleurs de noter que la plupart des intervenants du film appartiennent aux classes moyennes qui sont, dans l'optique du néo-libéralisme, vouées à l'extinction. On pourrait dire à propos du film de Lanctôt qu'il représente bien leur chant du cygne et citer à leur sujet les mots de Viviane Forrester:

Masse des utilisés, si vite inutiles et qui, chômeurs, se conçoivent superflus, car l'imposture veut que soit valorisé ce qui fait vivre et non la vie vécue - la vie qui les habite, et leurs corps vivants manifestant cette vie. Seul vaut ce qu'on paie. On paie l'aliénation. La vie qu'on vous prend. Pas cher. (...) Agitation bénie. Être reconnu, rémunéré (bien ou mal, peu importe), l'essentiel c'est d'être supprimé, comme son prochain, avec lui, près de lui. La foule, chacun des éléments de cette foule, joue le rôle qui lui est assigné: celui de ne pas investir le sien. Vaste conjuration, généreuse entreprise. Annulez-vous les uns les autres. Protégez-nous de nous.

De notre différence. Supprimez le présent (p. 20). 
Le film présente un système fatal et non le système réel. S'il témoigne de certains des effets aliénants du système sur les individus, c'est pour ne pas nommer ce système et rester au plan anecdotique, narratif. Pour tirer toutes les conséquences du système tel qu'il existe, il faut encore une fois se replonger dans le réel. C'est l'un des avatars de notre époque et de notre société que de ne plus pouvoir nommer le capitalisme réel comme le grand coupable. On préfère ici se complaire dans le discours du désenchantement nationaliste qui est indissociable d'une génération gâtée et vieillissante; une génération dont les acquis économiques sont mis en péril par la crise. Une génération qui craint par-dessus tout de «disparaître», à l'heure où ses propres enfants et les grandsparents de ses enfants cherchent à survivre moralement et physiquement à travers l'implacable rigueur des lois économiques. Deux films de la série me semblent témoigner plus spécifiquement de ce désenchantement et du chantage démographique qui lui est concomitant: Le Voyage au bout de la route ou La Ballade du pays qui attend (1987) de Jean-Daniel Lafond et Voyage en Amérique avec un cheval emprunté (1987) de Jean Chabot.

Se situant d'emblée dans la quête du Saint-Graal nationaliste, il est significatif que ces deux films adoptent en quelque sorte la forme du «travelogue». Leur différence essentielle réside dans la direction choisie pour atteindre le même but. Le film de Lafond constitue un voyage vers l'intérieur, vers l'arrière-pays, vers le Nord. Un nord mythique puisque l'idée dominante du film réside dans le pays imaginaire, celui des chansonniers. Partant de l'idée vraie mais lassante dans sa répétition (voire complaisante) du «rêve québécois qui est passé par la chanson», on a encore une fois droit à l'inflation verbale sur Félix Leclerc et à la focalisation sur le Français de service, Jacques Douai - sorte de caution culturelle ambulante. Le film débute à Paris, atterrit à Mirabel, passe par la banlieue, séjourne à Québec et nous entraîne sur la Côte-Nord jusqu'au bout du système routier pavé. La ville de Montréal est esquivée. Elle n'existe plus. Il faut dire que Montréal ne concorde pas avec la vision nostalgique d'une société tricotée serrée que le film pose comme antithèse à la réalité québécoise actuelle où les maillons traditionnels se défont. D'ailleurs, le regard du réalisateur, relayé par Jacques Douai, manifeste cette incompréhension profonde des enjeux du Québec contemporain. Il est significatif que Lafond confie à un Français le rôle de l'étranger qui découvre le pays; ainsi le regard de l'autre n'est-il pas trop loin du même. L'aurait-il confié à un citoyen ou une citoyenne des dizaines de minorités culturelles du Québec? Peut-être aurait-il ainsi découvert de nouvelles manières de circuler sur la terre et donc d'envisager ce territoire, peut-être aurait-on une idée moins 
sombre de l'avenir. Car le film participe de cette nostalgie de certains intellectuels québécois où l'unanimisme d'un passé radieux ne sait pas comment envisager le présent, pas plus qu'il ne saurait anticiper l'avenir. C'est ce refus d'investir le présent et la réalité du présent qui est à l'origine du grand silence des formes du passé où ils se complaisent. C'est un silence fatal au moment où il faudrait la mort du silence. Car, comme l'écrit encore Forrester: «(...) c'est peut-être là, (....) qu'on retrouve l'Histoire (...); non pas dans le silence de la mort, mais dans la mort du silence d'où déferleraient les langages, les clameurs nombreuses, tout ce que la langue, l'Histoire ont fait taire; langages de notre histoire, de nos histoires, de ce que nous pourrions être» (p. 34-35).

Dans Voyage en Amérique avec un cheval emprunté, l'enfant à naître est l'occasion pour Jean Chabot d'un retour «quelque part, du côté du souffle originel et de la vérité profonde des choses». Cette volonté de retour aux origines et la forme testamentaire que prend ici le voyage, trouvent leur raison d'être dans le désenchantement total de l'auteur et de l'«insidieuse menace» de la disparition de la race. L'auteur pose d'ailleurs la question en ces termes: «Que reste-t-il de cette race audacieuse dans le bel aujourd'hui?» Polarisé entre «la mort du père» et «la vie qui fait signe», Voyage en Amérique cherche à projeter l'individu à la grandeur du continent. C'est le scénario américain par excellence qui repose sur l'ethnocentrisme et l'idéologie qui lui est concomitante, où «le domaine public se soumet au privé» ( $\mathrm{Paz}, \mathrm{p}$. 58). L'attention qu'accorde Chabot aux peuples aborigènes n'existe que pour prendre la mesure de la disparition de sa propre race: «des civilisations disparaissent par manque apparent de complexité.» Dans cette quête du sens où l'autobiographique donne la mesure au scénario de la catastrophe, il n'y a pas de demimesure dans l'appropriation des signes du fatal. Reposant sur une bande-son totalisante où la voix off de Gilles Renaud exerce une pression telle qu'elle fait sourdre des images de l'Amérique, un Québec sans l'autre, le film de Chabot, en se confinant dans les paramètres du «retour à l'origine» et de «l'innocence» de l'enfant à naître, ignore l'histoire pour se confiner au jugement d'ordre moral. Il n'est pas étonnant alors qu'on puisse entendre en sourdine l'introduction des valeurs de l'idéologie américaine dans la pensée de certains intellectuels québécois, dans la mesure où, comme l'explique Octavio $\mathrm{Paz}$, les intellectuels nord-américains «ont remplacé la vision historique par le jugement d'ordre moral» (p. 61). Et c'est pourquoi cette quête de l'autre ou plutôt chez l'autre, ressemble étonnamment au même, dans la mesure où elle participe de ce que j'ai appelé la vision unanimiste du passé québécois, et qu'en débordant sur le présent pour tenter de 
l'expliquer, celle-ci participe de ce puritanisme provincial qui a toujours été la source de l'isolationnisme américain et de sa ségrégation intérieure.

Et si cette peur bien réelle engendrée par la mondialisation des marchés et de la culture - mondialisation qui n'est pas plus américaine qu'elle n'est japonaise ou européenne, qui est celle des banques et des multinationales - mais qui s'exprime ici par la peur du géant américain, qui est un géant au pied d'argile, dissimulait mal la véritable peur québécoise qui est celle de l'autre, du différent? Et si derrière le discours nostalgico-nationaliste se profilait, par-delà son incapacité à appréhender le présent, le complexe de la fermeture et de l'isolationnisme? L'idéologie américaine ne nous aurait-elle pas gagnés de manière beaucoup plus perverse qu'on ne le croyait jusqu'à présent? D'où peut-être le discours démagogique sur le «retour du père» avec lequel certains intellectuels d'ici font leurs gorges chaudes; cette tentative pour contrer le soi-disant vide qui nous menace, pour le remplir de loi et de coercition et, comme l'écrit Forrester, "pour préférer le refuge offert par les systèmes coercitifs, qui donnent du plein, de l'objet, de la chose à désirer, à défaut de désir» (p. 24), alors que nous devrions plutôt «admettre qu'il n'y a pas de père vers qui faire retour. Admettre que nous ne sommes pas l'expression d'un désir. Qu'il n'y a, à la limite aucun désir. Ou encore aucun objet au désir sans fin qu'il y ait du désir» (p. 23-24).

Si Le Voyage au bout de la route et Voyage en Amérique avec un cheval emprunté constituent en quelque sorte des quêtes pour retrouver «le même», Alias Will James (1988) de Jacques Godbout et Le Grand Jack (1988) d'Herménégilde Chiasson présentent plutôt des personnages en quête de «l'autre». En fait, les deux derniers films de la série instaurent une perspective plus nuancée de l'«américanité», parce qu'ici l'enjeu de l'identité n'en est plus un d'analogie mais de différence. Dans le cas de Will James/Ernest Dufault, il repose sur la structure mythique du cow-boy et «des Québécois qui vivent tout naturellement au niveau du mythe.» D'où l'importance que revêt pour Will James le grand vide américain: c'est là qu'il peut, à l'image de la page blanche pour l'écrivain, inventer sa propre vie plutôt que de subir son existence. Parce que les événements font de lui un hors-la-loi, il quitte l'espace exigu où se déterminent, dans la contrainte, toutes les existences. Echappant en quelque sorte au discours du père, il peut dès lors entrer dans l'espace de la création. Mais il n'y échappe jamais totalement puisque le même, les origines, viendront constamment le hanter. De même Kerouac fuyant la ville parce que «les intellectuels des villes ne sont que des insensés tolérables qui ne savent vraiment pas comment continuer à vivre», cherchant 
«la grande chose pour laquelle on est sensé vivre; la grande foi», trouvant «dans la nature extatique des Noirs d'Amérique» et dans l'immobilité contemplative orientale les alternatives au grand silence mortifère dont l'Amérique est tout entière imprégnée. Ce jeu entre l'errance et l'immobilité, cette découverte de la liberté où il n'y a «pas de réponse tout juste un écho» (Forrester, p. 24), ce refus de toutes les formes de coercition, bref le mobile d'Ernest Dufault et de Jack Kerouac ne pouvait appartenir qu'à l'Amérique francophone et ils ne pouvait s'exprimer que par l'écriture. D'une part parce que leurs origines sont entièrement marquées par la différence, elle-même fondatrice de culture, et d'autre part parce que l'écriture est le lieu de la marginalité, de la différence, le lieu ultime où l'on peut «oublier l'oubli, afin de retrouver la mémoire et d'acquérir celle du présent, que censure l'Histoire» (Forrester, p. 26).

Au sein des films de la série «L'Américanité», je vois donc se développer une dispute entre la vision pessimiste et fatale du présent, conséquence de ce travail du deuil en conformité avec le discours du père, ce roman familial où «l'Histoire devient un récit truqué qui rafle le passé, sature l'avenir, refoule l'immédiat, qui ne relate pas le passé, mais l'altère, le châtre» et où «la fraude devient le synopsis de nos vies» (Forrester, p. 24-25), et le point de vue représenté par Will James et Jack Kerouac d'un travail du présent, voué à «la mémoire du présent. D'un présent qui survit à la mort, toute chronologie dissoute» (Forrester, p. 25). Travail du texte, travail de la différence qui peut témoigner de ce que la québécitude a à gagner de la pluralité. Ainsi pourrons-nous contrer le discours alarmiste du nata-fatalisme déterminé par la mort, la continuité, le narratif. «L'Amérique n'a pas compris le montage, l'Amérique reste narrative», écrivait Eisenstein; si notre différence n'est plus ce qui fonde notre rapport au présent, nous ne saurons pas distinguer les instances discursives de l'histoire; aveuglés par le même, nous ne saurons pas «dévisager l'imprévisible» et accueillir le différent.

\section{Université de Montréal}

\section{OUVRAGES CITÉS}

Eisenstein, Sergeï M. Mémoires. Paris: Julliard, 1989.

Forrester, Viviane. La Violence du calme. Paris: Éditions du Seuil, 1980.

Foucault, Michel. L'Ordre du discours. Paris: Gallimard, 1971.

Paz, Octavio. Une planète et quatre ou cinq mondes. Paris: Gallimard, 1985. 